Original Article

Journal of Epilepsy Research pISSN 2233-6249 / eISSN 2233-6257

Received February 5, 2020

Revised April 27, 2020

Accepted June 23, 2020

Corresponding author:

Kanij Fatema, FCPS

Department of Pediatric Neurology,

Bangabandhu Sheikh Mujib Medical

University, Shahbagh, Dhaka 1000,

Bangladesh

Tel. $+880-1713097751$

Fax. +880-1713097751

E-mail; maiomonami@gmail.com

\title{
Epilepsy in Children with Tuberous Sclerosis Complex: A Prospective Observational Study in Bangladesh
}

\author{
Md Mizanur Rahman, FCPS, Kanij Fatema, FCPS \\ Department of Pediatric Neurology, Bangabandhu Sheikh Mujib Medical University, Dhaka, Bangladesh
}

Background and Purpose: Epilepsy is an important neurologic feature of patients with tuberous sclerosis complex (TSC). Most common seizure types are focal seizure and epileptic spasm. Seizure control often requires multiple antiepileptic drugs. This study has been done to evaluate the seizure types, electroencephalography (EEG), neuroimaging features, and drug treatment of epilepsy in TSC.

Methods: This prospective observational study has been conducted on epilepsy patients with TSC at Bangabandhu Sheikh Mujib Medical University from 2011 to 2019.

Results: Seventy patients with a mean \pm standard deviation age of $5.64 \pm 3.96$ years were identified and $57.1 \%$ were female. Most common type of seizure was focal seizure (46\%). Epileptic spasm occurred in $17 \%$ of patients and all of them had seizure onset before 1 year. In $47 \%$ of patients EEG showed focal epileptic discharge; hypsarrhythmia was found in most of the patients with epileptic spasm. Majority of the patients needed more than one drug to control seizure. Only $34 \%$ of patients were seizure free for at least 12 months and $22.8 \%$ had drug resistant epilepsy.

Conclusions: This study highlights the pattern of seizure, treatment pattern, response to drug, and short-time outcome of children with TSC with epilepsy in a developing country like Bangladesh. (2020;10:18-23)

Key words: Child, Tuberous sclerosis, Epilepsy

\section{Introduction}

Epilepsy is an important neurologic manifestation of patients with tuberous sclerosis complex (TSC). The reported prevalence of epilepsy in TSC is about $62 \%$ to $93 \%{ }^{1-3}$ Epilepsies associated with TSC usually present during infancy and early childhood, but it can also occur at any age. The most common type of seizures are focal seizure and epileptic spasm. However, the pattern of seizures in TSC changes over time. About half of the patients have refractory epilepsy. ${ }^{4.9}$ There are limited numbers of studies performed on TSC in developing countries. Thus, this study was done in children with TSC with epilepsy to evaluate the types of seizure, electroencephalography (EEG) features, neuroimaging pattern, drug treatment and response to treatment.

\section{Methods}

\section{Study protocol}

This study was carried out at a tertiary care center in Bangladesh. It was conducted on patients with TSC and epilepsy, children of all age
(0-18 years), were included during the period of January 2011 to October 2019. Patients were diagnosed as a case of TSC according to TSC clinical diagnostic criteria. ${ }^{10}$ initially, 84 patients were included, the data of seventy patients were ultimately used as adequate data for fourteen patients were missing. It was an observational study.

Detail history regarding seizure semiology, family history, developmental status, treatment history were taken. Formal clinical examination with special emphasis on eye, skin, abdomen, chest, nervous system, developmental assessment and psychological evaluation were performed. Neurobehavioural disorders like autism spectrum disorder and attention deficit hyperactivity disorder were diagnosed with Diagnostic and Statistical Manual V (DSM V). Intellectual disability was diagnosed when IQ was less than 70. The tools used here were Bayley Scales of Infant Development III, Wechsler Preschool and Primary Scale of Intelligence III, and Wechsler Intelligence Scale for Children-Revised IV.

For all patients a neuroimaging, preferably magnetic resonance imaging (MRI) of brain, and EEG were conducted. For target organ screening, color Doppler echo, USG of whole abdomen, X-ray chest, formal ophthalmological evaluation were performed. Patients were 
followed up 6-monthly for a period of at least 1 year.

\section{Ethical implication}

Informed written consent was obtained from the parents or legal guardians of all participants. The study protocol was approved from the Internal Review Board of Bangabandhu Sheikh Mujib Medical University.

Table 1. Demographic and clinical characteristics of the study subjects $(n=70)$

\begin{tabular}{lc}
\hline Demographic characteristic & Value \\
\hline Age (years) & $5.64 \pm 3.96(1-16)$ \\
Sex & $40(57.1)$ \\
Female & $30(42.9)$ \\
Male & \\
Developmental status & $30(42.9)$ \\
Normal & $39(54.3)$ \\
Developmental delay & $2(2.9)$ \\
Developmental regression & \\
Family history of TSC & $5(7.1)$ \\
Present & $65(92.9)$ \\
Absent &
\end{tabular}

Values are presented as mean \pm standard deviation (range) or number (\%).

TSC, tuberous sclerosis complex.

\section{Data collection and analysis}

Data was analyzed by Statistical Package for Social Sciences (SPSS-22 version) software (SPSS Inc., Chicago, IL, USA).

\section{Results}

\section{Patient demographics}

In this study seventy patients were analyzed. Mean age of the patients was $5.64 \pm 3.96$ years. Among them 62 patients $(88.6 \%)$ were bellow or equal to 10 years old. There was slight female predominance $(57.1 \%)$ among the patients. Only $7.1 \%$ of patients had positive family history of TSC (Table 1).

\section{Clinical manifestations of TSC}

Regarding skin manifestation, all the children had skin manifestations; most common finding was ash leaf spot (74\%). Other skin findings were adenoma sebaceum (60\%), shagreen patch (33\%), café au-lait spot (4.28\%), and subungual fibroma (Fig. 1). Most of the patients had multiple types of skin findings. Apart from neurological findings and skin manifestations, other systems involvement was minimal, namely four patients with cardiac involvement (rhabdomyoma and tricuspid valve regurgitation), two patients with renal involvement (angiomyolipoma and renal cyst), one patient with oral fibroma, and two patients with cataract. No patient was found with chest finding (Table 2).
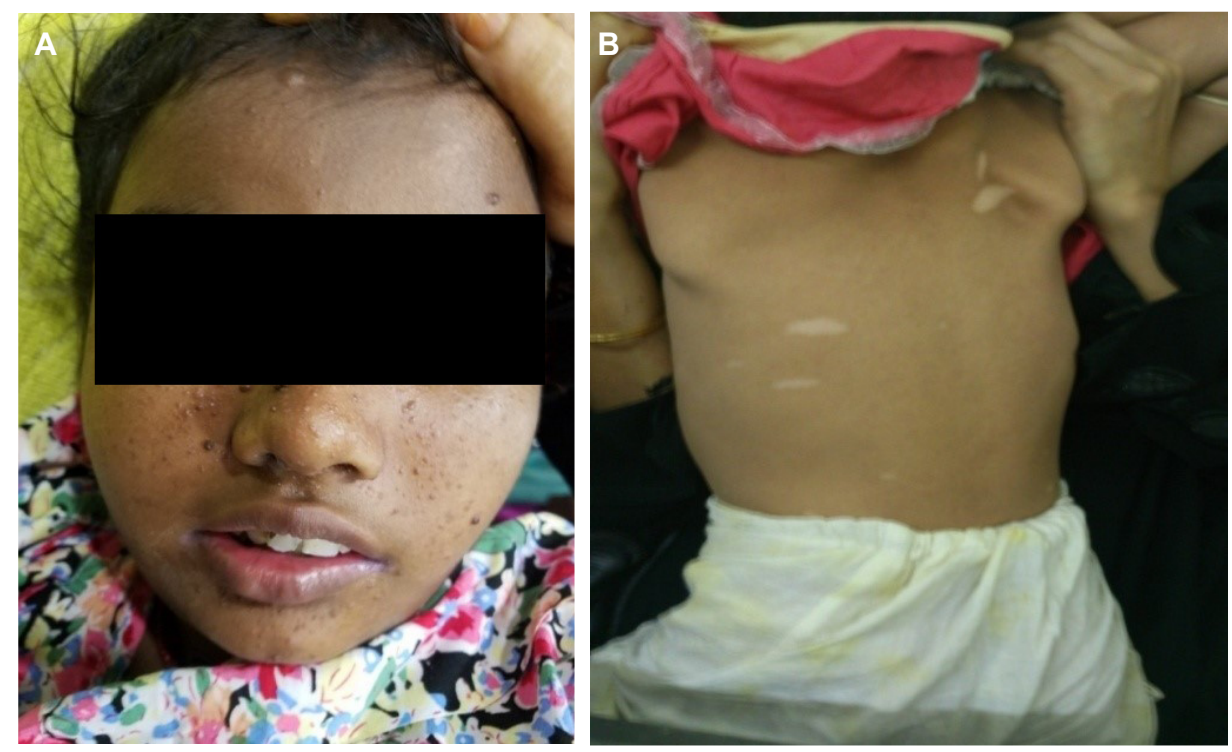

Figure 1. (A) Adenoma sebaceum in a patient with tuberous sclerosis complex. (B) Ash leaf spot in skin. 
Table 2. Physical features of the study subjects $(n=70)$

\begin{tabular}{lc}
\hline Physical feature & Value \\
\hline Skin manifestations & \\
Ash leaf (hypopigmented macule) & \\
Adenoma sebaceum & $42(60.0)$ \\
Shagreen patch & $23(33.0)$ \\
Café au-lait spot & $3(4.3)$ \\
Subungual fibroma & $1(1.4)$ \\
Organ involvement & \\
Heart & $4(5.7)$ \\
Kidney & $2(2.9)$ \\
Eye & $2(2.9)$ \\
Gastrointestinal & $1(1.4)$ \\
\hline
\end{tabular}

Values are presented as number (\%).

Table 3. Distribution of the study subjects by seizure criteria, EEG and follow up $(n=70)$

\begin{tabular}{lc}
\hline & Value \\
\hline Type of seizure & $12(17.1)$ \\
Epileptic spasm & $32(45.7)$ \\
Focal & $19(27.1)$ \\
Generalized & $4(5.7)$ \\
Other & $3(4.3)$ \\
Epileptic spasm followed by focal & \\
Age of onset & $26(37.1)$ \\
$<6$ months & $16(23.0)$ \\
$6-12$ months & $10(14.0)$ \\
$1-5$ years & $18(25.7)$ \\
$>5$ years & \\
EEG & $33(47.0)$ \\
Focal discharge & $17(24.0)$ \\
Generalized discharge & $10(14.3)$ \\
Hypsarrhythmia & $8(11.4)$ \\
Epileptic encephalopathy (other than LGS) & $2(2.85)$ \\
LGS & \\
Follow up & $24(34.2)$ \\
Seizure free for 12 months & $27(38.5)$ \\
Seizure controlled for 3 months & $16(22.8)$ \\
Drug resistant epilepsy & $3(4.2)$ \\
Relapse &
\end{tabular}

Values are presented as number (\%).

EEG, electroencephalography; LGS, Lennox-Gastaut syndrome.

\section{Seizure in studied patients (Table 3)}

\section{Onset of seizure}

Thirty-seven percent of the patients had the onset of seizure in the first 6 months of life. About 26 percent (25.7\%) of patients had the
Table 4. Neuroimaging findings of the study subject $(n=70)$

\begin{tabular}{lc}
\hline Neuroimaging finding & Value \\
\hline SEN+cortical tuber & $35(50.0)$ \\
SEN & $17(24.3)$ \\
Cortical tuber & $15(21.4)$ \\
SEN+arachnoid cyst & $1(1.4)$ \\
SEGA+SEN & $1(1.4)$ \\
SEN+cortical tuber+arachnoid cyst & $1(1.4)$ \\
\hline
\end{tabular}

Values are presented as number (\%).

SEN, subependymal nodule; SEGA, subependymal giant cell astrocytoma.

onset of seizure after 5 years of age. Thus, in most of the patients, seizure onset was in first year of life (60\%) (Table 3).

\section{Type of seizure}

The most prevalent type of seizure was focal seizure occurring in about $46 \%$ of patients. Epileptic spasm occurred in $17 \%$ of patients and generalized seizure in $27 \%$ of patients. However, $4 \%$ of patients had a history of epileptic spasm followed by focal seizure and $5.7 \%$ of patients had other types of seizure. All the patients who had epileptic spasm presented in less than 1 year of age (Table 3).

\section{Developmental status of the studied children}

Regarding developmental status, more than half of the children $(54.3 \%)$ had developmental delay in at least one domain and thirty children $(42.9 \%)$ had normal development. While two patients (2.9\%) had regression of development (mainly cognition and motor), both of them had intractable epilepsy (Table 1).

\section{Electroencephalographic findings}

All the patients with epilepsy had abnormal EEG. Focal epileptic discharge was the most prevalent EEG change in this study group (47\%). Hypsarrhythmia was found in ten patients (two classical and eight modified hypsarrhythmia). While 17 patients (24\%) had generalized discharge, eight patients (11.4\%) had epileptic encephalopathy and two patients had the EEG suggestive of Lennox-Gastaut syndrome (Table 3).

\section{Neuroimaging findings}

Neuroimaging (computed tomography scan or MRI of brain) were performed in all the studied subjects. 50\% (35) of patients had both subependymal nodules (SEN) and cortical tubers, while 24\% (17) had only SEN and 21\% (15) had only cortical tubers. One patient had 

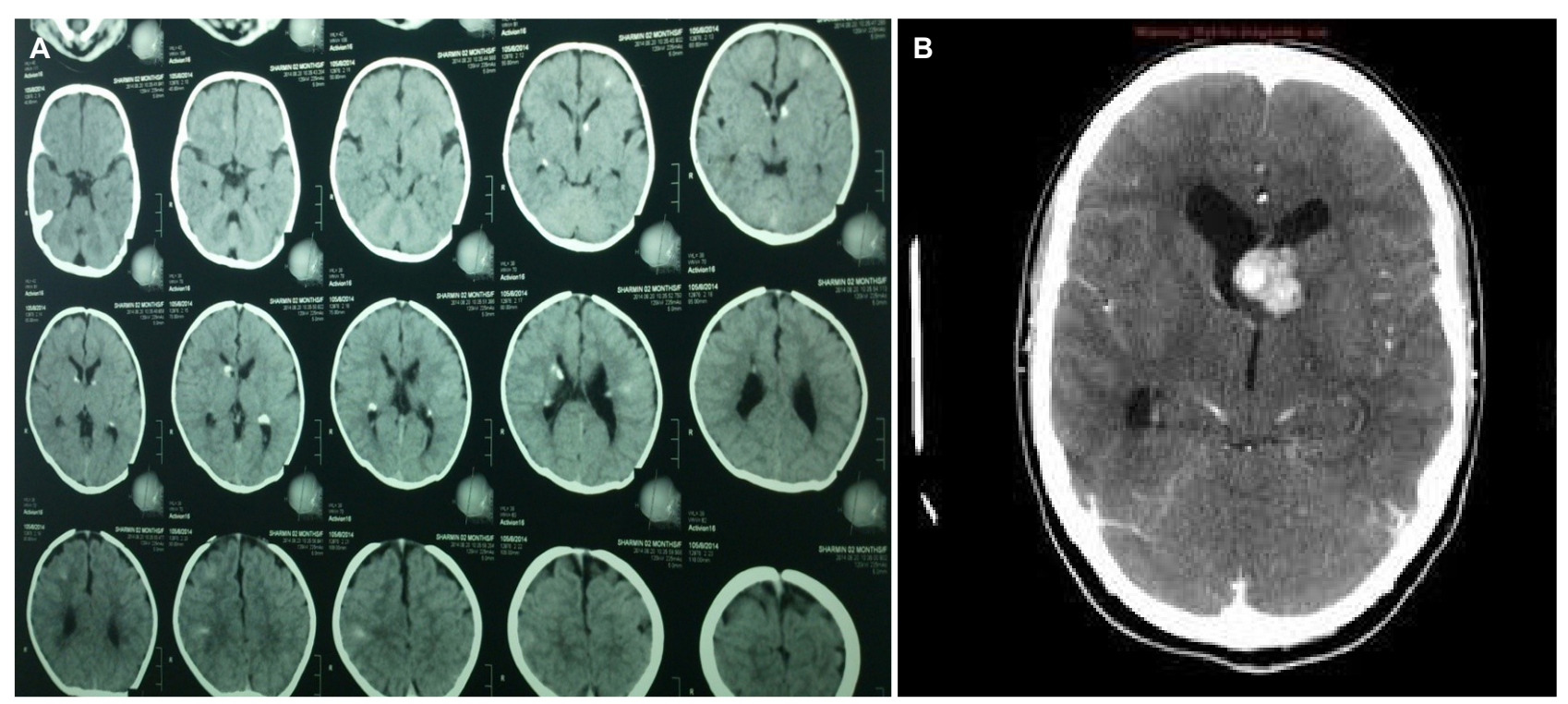

Figure 2. (A) CT scan of brain: multiple subependymal nodules. (B) CT scan of brain showing subependymal giant cell astrocytoma. CT, computed tomography.

Table 5. Antiepileptic drugs used in study subjects $(n=70)$

\begin{tabular}{lc}
\hline & Value \\
\hline Number of drugs & \\
One drug & $24(34.0)$ \\
Two drugs & $18(26.0)$ \\
Three drugs & $14(20.0)$ \\
Four drugs & $11(16.0)$ \\
Five drugs & $3(4.0)$ \\
Drugs used & \\
Vigabatrin & $39(55.7)$ \\
Sodium valproate & $33(47.0)$ \\
Levetiracetum & $19(27.0)$ \\
Phenobarbitone & $11(15.7)$ \\
Carbamazepine & $9(13.0)$ \\
Benzodiazepines & $9(13.0)$ \\
Oxcarbazepine & $8(11.0)$ \\
Prednisolone & $2(3.0)$ \\
Topiramate & $2(3.0)$ \\
ACTH & $1(1.5)$ \\
\hline
\end{tabular}

Values are presented as number (\%).

$\mathrm{ACTH}$, adrenocorticotropic hormone.

subependymal giant cell astrocytoma (SEGA) along with SEN. Two other patients had multiple central nervous system findings; one patient had both SEN and temporal cyst while another patient had SEN, cortical tuber and cyst. No patient had white matter abnormality or hydrocephalus (Table 4, Fig. 2).

\section{Antiepileptic drugs (AEDs) used}

Regarding AED use, more than half of the patients (55.7\%) received vigabatrin either as a single agent or in combination with other drugs. Among these patients taking vigabatrin, 16 patients had epileptic spasm (ES). In this cases, vigabatrin was started as the first or second line drug. Eight patients with IS completely responded to vigabatrin while three patients had partial response and in one patient vigabatrin was withdrawn due to somnolence. In this patients injection adrenocorticotropic hormone (ACTH) was given. Twenty six percent of children in this study group got two AED, 20\% got three drugs, $16 \%$ got four AED and $4 \%$ got five AED. In our cohort, $34.2 \%$ of patients were seizure free for 12 months, $22.8 \%$ had drug resistant epilepsy, $38.5 \%$ were seizure free for at least 3 months with 1 or 2 drugs, and $4.2 \%$ had relapse (Table 5).

\section{Treatment with mammalian target of rapamycin (mTOR) antagonist}

Fifteen patients (21.4\%) of this study group got mTOR antagonist (everolimus) for a period of 2 months to 1 year. Out of this, six patients were seizure free, but it was an add-on with other AEDs. Out of 15 patients, one patient had SEGA which showed decrement of size after treatment. 


\section{Short term outcome of the studied children}

In follow-up, 24 patients (34.2\%) were seizure free for at least 1 year, 16 patients (22.8\%) had ongoing seizure and 27 patients (38.5\%) had epilepsy controlled for at least 3 months with drug. Three patients had epilepsy relapse (Table 3).

\section{Discussion}

This study is a longitudinal observational study of children with TSC with epilepsy in a developing country like Bangladesh. Here all the patients were managed at a tertiary care centre. This study will contribute in better understanding of epilepsy and other co-morbidities in children with TSC along with the pattern of EEG and unique neuroimaging findings. This study has been performed in 70 patients who have TSC and epilepsy with mean age of $55.64 \pm 3.96$ years.

Although epilepsy is not diagnostic criteria of TSC, it is an important neurological feature. The prevalence of epilepsy in TSC is as high as $90 \% .{ }^{1,11}$ In this study group the most prevalent form of seizure was focal seizure. In about $46 \%$ of children, focal seizure was the initial pattern of seizure while in $4.3 \%$ of patients, focal seizure occurred after epileptic spasm. This finding coincides with the study done by Devis et al., ${ }^{12}$ who found focal seizure in $55 \%$ of patients. They found both focal seizure and $\mathrm{IS}$ in about $36 \%$ of patients, which is quite higher from our study finding. ${ }^{12}$ In a TuberOus SClerosis registry to increase disease Awareness (TOSCA) study also, focal seizure was the most prevalent type of seizure occurring in $67.5 \%$ of patients. ${ }^{13}$

Generalized seizure was the second most common type of seizure in this study group occurring in about $27 \%$ of children. Among them, $13(68 \%)$ had focal onset. Remaining six children (32\%) had true primary generalized epilepsy. A similar study done by Chu-Shore et al. ${ }^{1}$ revealed that about $37 \%$ of patients had generalized seizure although most of them had focal onset like our study. They also suggested that the number of patients with true generalized epilepsy in TSC may be overestimated as here the categorization has been performed based upon the clinical description, not the ictal EEG recording. As TSC neuropathogenesis favors the focal onset of the seizure, it is most likely that most of the seizures are partial in onset with or without secondary generalization. ${ }^{1}$

In this cohort, $17 \%$ of patients had epileptic spasm and this is a bit lower than the prevalence of epileptic spasm in a TOSCA study and a study done by Chu-Shore et al., ${ }^{1}$ where the overall prevalence is about $38.9 \%$ and $37 \%$, respectively. ${ }^{13}$ The occurrence of epileptic spasm is higher in early ages, particularly in the first 2 years which is about $46 \%$ of patients in some studies. ${ }^{14}$

Usually in TSC, the onset of seizure is in early childhood, mostly in infancy, although they can occur at any age. Moreover, patients remain at risk of epilepsy, particularly focal epilepsy, throughout their lifetime. ${ }^{12}$ In our cohort, 37\% of patients had their first seizure before 6 months and 23\% had it between 6 to 12 months. Thus, the onset of seizure bellow 1 year was $60 \%$. However, delayed onset seizure was not uncommon. About $25.7 \%$ of patients had their first seizure after 5 years of age. Our study result thus matches with that of TOSCA study, where $79 \%$ of patients had their first seizure before 2 years. They also noticed the late onset of seizure even at the age of 40 years. In this study group, they have noticed that occurrence of IS was higher in less than 2 years, but the occurrence rate of focal seizure was equal in both early and late onset seizure. ${ }^{13}$

EEG plays an important role for diagnosis, prognosis and treatment of epilepsy in TSC. ${ }^{13}$ In our cohort, all the patients had abnormal EEG. Most common type of abnormality found are focal discharges arising from temporal and occipital areas. Some of them were multifocal in origin. In cases of epileptic spasm, 14.3\% had hypsarrhythmia and the rest of them have multifocal discharges.

The commonest neuroimaging finding was subependymal nodule (78.9\%), while other findings were cortical tuber in $21.4 \%$ of patients, SEGA in one patient, and arachnoid cyst in one patient. In a related study done by Davis et al., ${ }^{12}$ they found SEN in $90 \%$ of patient, both SEN and cortical tuber in $89 \%$ of patients, and normal neuroimaging in $4 \%$ of patient. In our cohort, we did not find any normal neuroimaging. It is here to mention that; neuroimgaing in TSC may initially appear normal and might found in follow up study. ${ }^{12}$

Meticulous choice of AED is very important in TSC. Seizure control in an early stage is closely related to better psychomotor outcome. ${ }^{1,14}$ In our cohort, $34.2 \%$ of patients went to remission, $22.8 \%$ had drug resistant epilepsy, $38.5 \%$ had epilepsy with 1 or 2 drugs, and $4.2 \%$ had relapse. Treatment with GABAergic drugs (i.e., vigabatrin) plays a vital role in TSC. The current recommendation is vigabatrin, which should be used as a first line AED treatment for epileptic spasm with TSC and for focal seizure before the age of 1 year. ${ }^{4,15}$ Almost all the patients with epileptic spasm received vigabatrin in our cohort, but as one patient developed Lennox-Gastaut syndrome, we did not gave him vigabatrin. Other drugs used here were Na valproate, levetiracetum, phenobarbitone, benzodiazepine, oxcarbazepine, carbamazepine, topiramate, and oral steroid etc. In one patient who had epileptic spasm and developed side effect of vigabatrin, injection ACTH was given. Seizure was controlled with the administration of 1 
or 2 drugs in most of the patients; very few patients needed more than 2 drugs. Drug resistant cases were $22.8 \%$, which has similarity with that of TOSCA study were, in epileptic spasm the resistant cases were $15.5 \%$, and in focal seizure it was $38 \%{ }^{16}$

The recent Examining Everolimus in a Study of Tuberous Sclerosis Complex (EXIST) 3 study shows higher efficacy of adjuvant everolimus therapy in patients with drug resistant seizure associated with TSC compared to placebo. ${ }^{17}$ Seizure relapse is not uncommon in TSC. In a study by Sparagana et al., ${ }^{18}$ more than one forth patients experiences relapse while Chu-Shore et al. ${ }^{1}$ found $9.1 \%$ of patients had epilepsy relapse. In our study it was much less, about 4.2\%. This discrepancy may be due to short time follow up and the difference of the population studied. ${ }^{1}$ However, it is importnant to taper AEDs in patients when the seizure is well controlled and improved or resolved EEG abnormalities. Although there are different studies regarding the ketogenic diet, surgery and vagus nerve stimulation in TSC with epilepsy, none of the modalities have been used in this study. ${ }^{17,19}$

Apart from epilepsy and neurological stigma, there is the involvement of multiple organs in TSC. The commonest cutaneous finding in this cohort was ash leaf spot (74\%). We also searched for target organ involvement and found that heart was most commonly affected in our cohort. Along with this, renal, eye, and oral involvement was detected in this cohort.

This prospective study demonstrates the pattern of seizure, EEG changes, neuroimaging findings of children with TSC with epilepsy. It also highlights the response to therapy. In early infancy common forms of seizure were epileptic spasm and focal seizure. It also confirms that epilepsy with TSC is difficult to treat as only $34.2 \%$ of patients had a sustained seizure freedom and $4.2 \%$ had relapse. Also, a good number of patients needed more than two drugs to control seizure.

\section{References}

1. Chu-Shore CJ, Major P, Camposano S, Muzykewicz D, Thiele EA. The natural history of epilepsy in tuberous sclerosis complex. Epilepsia 2010; 51:1236-41.

2. Jeong $A$, Wong M. Systemic disease manifestations associated with epilepsy in tuberous sclerosis complex. Epilepsia 2016;57:1443-9.

3. Krueger DA. Management of CNS-related disease manifestations in patients with tuberous sclerosis complex. Curr Treat Options Neuro/ 2013; 15:618-33.
4. Curatolo P, Jóźwiak S, Nabbout R; TSC Consensus Meeting for SEGA and Epilepsy Management. Management of epilepsy associated with tuberous sclerosis complex (TSC): clinical recommendations. Eur J Paediatr Neurol 2012;16:582-6.

5. Curatolo P, Moavero R, de Vries PJ. Neurological and neuropsychiatric aspects of tuberous sclerosis complex. Lancet Neuro/ 2015;14:733-45.

6. Rosser T, Panigrahy A, McClintock W. The diverse clinical manifestations of tuberous sclerosis complex: a review. Semin Pediatr Neuro/ 2006; 13: 27-36.

7. Cusmai R, Moavero R, Bombardieri R, Vigevano F, Curatolo P. Long-term neurological outcome in children with early-onset epilepsy associated with tuberous sclerosis. Epilepsy Behav 2011;22:735-9.

8. Humphrey A, MacLean C, Ploubidis GB, et al. Intellectual development before and after the onset of infantile spasms: a controlled prospective longitudinal study in tuberous sclerosis. Epilepsia 2014;55:108-16.

9. Vignoli A, La Briola F, Turner $K$, et al. Epilepsy in TSC: certain etiology does not mean certain prognosis. Epilepsia 2013;54:2134-42.

10. Northrup H, Krueger DA; International Tuberous Sclerosis Complex Consensus Group. Tuberous sclerosis complex diagnostic criteria update: recommendations of the 2012 linternational Tuberous Sclerosis Complex Consensus Conference. Pediatr Neurol 2013;49:243-54.

11. Yates JR, Maclean C, Higgins JN, et al. The Tuberous Sclerosis 2000 Study: presentation, initial assessments and implications for diagnosis and management. Arch Dis Child 2011;96:1020-5.

12. Davis $P E$, Filip-Dhima $R$, Sideridis $G$, et al. Presentation and diagnosis of tuberous sclerosis complex in infants. Pediatrics 2017;140:e20164040.

13. Kingswood JC, d'Augères GB, Belousova $E$, et al. TuberOus SClerosis registry to increase disease Awareness (TOSCA) - baseline data on 2093 patients. Orphanet I Rare Dis 2017;12:2.

14. Eisermann MM, Ville D, Soufflet $C$, et al. Cryptogenic late-onset epileptic spasms: an overlooked syndrome of early childhood? Epilepsia 2006;47: 1035-42.

15. Krueger DA, Northrup $\mathrm{H}$; International Tuberous Sclerosis Complex Consensus Group. Tuberous sclerosis complex surveillance and management: recommendations of the 2012 International Tuberous Sclerosis Complex Consensus Conference. Pediatr Neurol 2013;49:255-65.

16. Nabbout R, Belousova E, Benedik MP, et al. Epilepsy in tuberous sclerosis complex: findings from the TOSCA study. Epilepsia Open 2019;4:73-84.

17. French JA, Lawson JA, Yapici $Z$, et al. Adjunctive everolimus therapy for treatment-resistant focal-onset seizures associated with tuberous sclerosis (EXIST-3): a phase 3, randomised, double-blind, placebo-controlled study. Lancet 2016;388:2153-63.

18. Sparagana SP, Delgado MR, Batchelor LL, Roach ES. Seizure remission and antiepileptic drug discontinuation in children with tuberous sclerosis complex. Arch Neurol 2003;60:1286-9.

19. Overwater IE, Bindels-de Heus $K$, Rietman $A B$, et al. Epilepsy in children with tuberous sclerosis complex: chance of remission and response to antiepileptic drugs. Epilepsia 2015;56:1239-45. 